\title{
METÁSTASIS DE CARCINOMA DE PRÓSTATA EN URETRA: A PROPÓSITO DE UN CASO
}

\author{
A. BLANCO DÍEZ, M. RUIBAL MOLDES, J. RODRÍGUEZ-RIVERA GARCÍA, \\ E. FERNÁNDEZ ROSADO, L. ÁLVAREZ CASTELO, F. GÓMEZ VEIGA, \\ M. GONZÁLEZ MARTÍN
}

Servicio de Urología. Hospital Universitario Juan Canalejo. La Coruña.

Actas Urol Esp. 27 (9): 735-738, 2003

\section{RESUMEN}

METÁSTASIS DE CARCINOMA DE PRÓSTATA EN URETRA: A PROPÓSITO DE UN CASO

Las metástasis peneanas de cáncer de próstata son raras, sobre todo en uretra y cuerpo esponjoso, aportamos un caso a la literatura. Se trata de un paciente con cáncer de próstata hormonorresistente que durante la evolución del mismo cursa con dificultad miccional y hematuria ocasional. Se observa durante la RTU unas formaciones mamelonadas en uretra peneana que se resecan, resultando ser metástasis de su carcinoma de próstata. El paciente se encuentra con buen estado general y sin clínica urinaria tras 7 meses de seguimiento. Nos parece muy interesante porque existen pocos casos reportados de metástasis en dicha localización.

PALABRAS CLAVE: Cáncer de próstata. Metástasis. Uretra.

\section{ABSTRACT}

PROSTATE CARCINOMA METASTATIC TO THE URETHRA: A CASE REPORT

Penile metastases from prostate carcinoma are uncommon events, much more in urethra and corpus spongiosum, we add a case to the preexisting literature. A patient with hormone resistant prostate cancer consults for haematuria and voiding difficulties. During TURP we observe tumors at the penile urethra wich are resected and result to be prostate cancer metastases. Patient is fine and without voiding disturbance after 7 months of following. We think is an interesant case because ther is few reports in literature.

KEY WORDS: Prostate cancer. Metastases. Urethra.

$\mathrm{L}$ as metástasis peneanas son raras en la práctica clínica, correspondiendo casi siempre a tumores vesicales, prostáticos, renales, rectales o testiculares. Los carcinomas prostáticos serían responsables del $28 \%$ de dichas metástasis. La localización más frecuente sería el prepucio y los cuerpos cavernosos siendo raras en uretra y cuerpo esponjoso ${ }^{1-5}$.

\section{CASO CLÍNICO}

Varón de 54 años de edad, fumador de 30 cigarrillos día. Consulta por primera vez en Uro- logía en mayo de 2000, por cuadro de infección urinaria baja y cólico nefrítico izquierdo.

Al tacto rectal se palpa una próstata fija, pétrea, e irregular, en ambos lóbulos. La ecografía no muestra alteraciones de interés, excepto un crecimiento prostático para la edad (próstata de aproximadamente 65 gramos). El PSA es de 223.

La biopsia de próstata detecta 6 cilindros marcadamente infiltrados por un adenocarcinoma Gleason VII. Se realiza gammagrafía ósea de estadiaje que no muestra datos de patología 
ósea, iniciándose bloqueo androgénico completo con antiandrógenos y análogos LH-RH (agosto de 2000).

Durante el seguimiento las cifras de PSA van ascendiendo de forma progresiva (enero 2001: 0.5, agosto 2001: 0.7, febrero 2002: 6.4, abril 2002: 14), sin que el paciente note empeoramiento de su estado general.

En febrero y abril de 2002 acude a urgencias por dos episodios de hematuria y retención de orina, siendo preciso sondaje vesical durante un mes. Progresivamente va empeorando su clínica obstructiva en dos meses llegando a orinar casi por goteo en junio de 2002. El estudio flujométrico en mayo de 2002 muestra un flujo máximo de $2 \mathrm{ml} / \mathrm{seg}$, con residuo de $80 \mathrm{ml}$. Debido a este empeoramiento claramente obstructivo de su enfermedad se le propone resección transuretral de la próstata.

En julio de 2002, cuando se introduce el resector para efectuar la resección de la próstata, se observa una uretra peneana repleta de formaciones mamelonadas de 0,5-1 cm, polipoideas, que dificultan la entrada hasta vejiga, debido a que reducen el calibre uretral y sangran al roce (Fig. 1). El resto de uretra bulbar y prostática es normal, algo desestructurada ésta última. No se realiza R.T.U. por difícil visión.

En julio de 2002 tras constatar un PSA de 22 , se procede a la retirada de antiandrógenos, manteniendo los análogos e incluyendo al paciente en un ensayo con Atrasentan (MOO244).

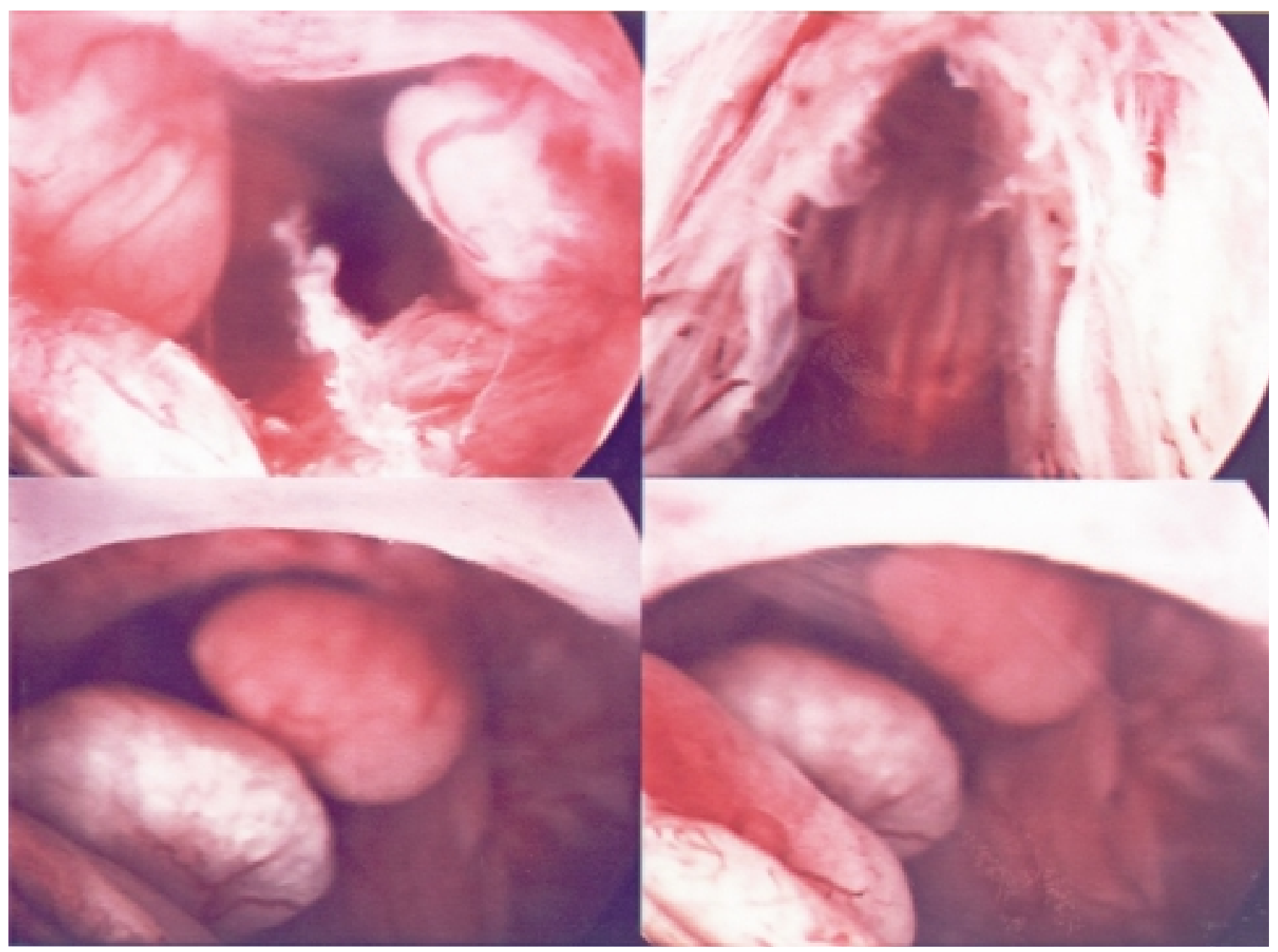

FIGURA 1. Se observa la uretra peneana repleta de unas lesiones de superficie lisa mamelonadas, y obstructivas, muy vascularizadas y de fácil sangrado. Finalmente resultaron ser metástasis uretrales a distancia del tumor prostático. 
La persistencia de severa clínica obstructiva justificó una resección de los mamelones uretrales y de la próstata, enviándose ambas muestras por separado al departamento de anatomía patológica para obtener la filiación histológica de los primeros. Se le mantuvo sondaje vesical durante 3 semanas. El diagnóstico histológico del tejido uretral fue: masas formadas exclusivamente por un adenocarcinoma Gleason X. En lo que se refiere al tejido prostático propiamente dicho, el diagnóstico fue el mismo.

En el momento actual el paciente se encuentra en un excelente estado general acudiendo a las consultas pertinentes según manda el protocolo del ensayo, sin sintomatología derivada de su enfermedad.

\section{DISCUSIÓN}

Es llamativo, teniendo en cuenta la importante vascularización del pene, la escasez de casos reportados de metástasis en el mismo. La primera gran revisión de la literatura mundial al respecto la realiza Abeshouse ${ }^{5}$ en 1961. Recopila en total 140 casos de metástasis peneana, de los cuales sólo 39 corresponden a carcinoma de próstata. La primera revisión de la literatura nacional la realiza Gómez Vegas recopilando 23 casos en 1991, de las que sólo 4 eran secundarias a carcinoma de próstata ${ }^{2}$. Sin embargo la mayoría de los casos reportados en la literatura se refieren a implantes en cuerpos cavernosos o prepucio, habiendo sólo 12 de metástasis puramente uretrales o de cuerpo esponjoso ${ }^{3,4,6-10,12}$.

Pueden existir varios mecanismos de aparición de metástasis peneanas descritos por Paquin en $1956^{11}$ :

- Extensión directa. A través de la orina que implantaría células tumorales de la próstata en la uretra.

- Implante tras manipulación prostática (por ejemplo resección transuretral) ${ }^{10,12,13}$, prostatectomía radical ${ }^{13}$.

- Embolismo arterial.

- Diseminación venosa.

- Diseminación linfática.

Es evidente que debido a las conexiones existentes entre el plexo venoso prostático y la vena dorsal del pene, la extensión por vía venosa retrógrada o linfática parece explicar bien la propagación de células desde la próstata al pene $^{5}$. En el caso que aportamos, la vía más probable habría sido la primera dado que no existía manipulación previa, aún así nuestros límites en el conocimiento de la patobiología tumoral no nos permite asegurar estas afirmaciones.

Las manifestaciones clínicas suelen consistir en hematuria asociada a sintomatología obstructiva secundaria al crecimiento intrauretral que condiciona micción entrecortada, micción por goteo o retención urinaria, en ocasiones asocia incapacidad para dirigir el chorro miccional.

En lo que atañe al estudio, no se diferencia del que exige cualquier clínica de dificultad miccional o hematuria. Hay que realizar uretrocistoscopia y uretrocistografía que suelen darnos imágenes características $^{1,13}$. En la cistouretrografía veríamos defectos de replección múltiples en uretra y en la uretroscopia neoformaciones mamelonadas intrauretrales. El estudio histológico requiere técnicas de inmunohistoquímica para determinar origen prostático o uretral.

El pronóstico es distinto según la metástasis sea en cuerpo cavernoso o en uretra y cuerpo esponjoso. Las metástasis en cuerpo cavernoso no tienen una supervivencia superior a los 12 meses $^{3}$, mientras que las segundas tienen mejor pronóstico, incluso con buena respuesta a tratamiento hormonal ${ }^{1,4,5,10}$.

En lo que se refiere al tratamiento, hay que tener en cuenta dos conceptos: por una parte que es un cáncer de próstata metastásico y/o localmente avanzado, lo cual tiene su tratamiento específico; en segundo lugar el problema miccional obstructivo del paciente. Las opciones que se pueden barajar serían: resección transuretral de las masas, como el caso descrito, con el consiguiente tratamiento hormonal o quimioterápico del tumor metastásico, administrar solamente tratamiento hormonal ${ }^{1,3}$, o en casos de metástasis en cuerpos cavernosos la penectomía o uretrectomía, siempre que no exista afectación de pelvis, o datos de enfermedad diseminada ${ }^{4,13}$. Algún grupo ha publicado el tratamiento con braquiterapia del carcinoma prostático metastásico en pene ${ }^{9,14}$. En cualquier caso el pronóstico parece sombrío aunque a medio-largo plazo. 


\section{REFERENCIAS}

1. LLARENA IBARGUREN R, VESGA MOLINA F, ACHA PÉREZ M, BLASCO VILLALONGA M, PERTUSA PEÑA C.: Metástasis uretrales por adenocarcinoma prostático. Arch Esp de Urol 1993; 46: 779-782.

2. GÓMEZ VEGAS A, SILMI MOYANO A, BLÁZQUEZ IZQUIERDO $J$ y cols.: Metástasis peneanas. Aportación de tres nuevos casos y revisión de la literatura nacional. Arch Esp de Urol 1991; 49: 10631067.

3. IVERSON AP, BLACKARD CE, SCHULBERG VA. Carcinoma of the prostate with urethral metastases. $J$ Urol 1972; 108: 901-904.

4. KOH E, SEKII K, FUJIOKA H et al.: Urethral metastasis from prostatic carcinoma as diagnosed by immunoperoxidase technique using prostate-specific antigen and prostate-specific acid phosphatase. Eur Urol 1987; 13: 142-144.

5. ABESHOUSE BS, ABESHOUSE GA.: Metastatic tumors of the penis: a review of literature and a report of two cases. J Urol 1996; 86: 99-112.

6. ELKIN M, MUELLER HP.: Metastasis from cancer of the prostate. Autopsy and roentgenocological findings. Cancer 1954; 7: 1246-1249.

7. KOTECHA N, GENTILE RL.: Carcinoma of prostate with urethral metastasis. Urology 1974; 3: 85-86.

8. SUBUDHI CL, PANDA SN, PANIGRAHI KK.: Urethral metastases from carcinoma of prostate. Indian $J$ Cancer 1994; 31: 31-33.
9. DE PORRE P.: Urethral metastases of a prostate cancer. A case report. Strahlenther Onkol 1993; 169: 256-257.

10. TAYLOR GB, MC NEAL JE, COHEN RJ.: Intraductal carcinoma of the prostate metastatic to the penile urethra: a rare demonstration of two morfologic patterns of tumor growth. Pathology 1998; 30: 218-221.

11. PAQUIN AJ, ROLAND SI.: Secondary carcinoma of the penis. Cancer 1956; 9: 626-630.

12. METHA SS, LEE JA, CHAPPLE CR.: Urethral metastases after transurethral resection of a malignant prostate. BJU Int 2001; 87: 126.

13. KOBAYASHI T, FUKUZAWA S, OKA H, FUJIKAWA K, MATSUI Y, TAKEUCHI H.: Isolated recurrence of prostatic adenocarcinoma to the anterior urethra after radical prostatectomy. $J$ Urol 2000; 164: 780.

14. GRIFFIN JH, WHEELER JS Jr, OLSON M, MELINA E.: Prostate carcinoma metastatic to the penis: magnetic resonance imaging and brachytherapy. $J$ Urol 1996; 155: 1701-1702.

Dr. A. Blanco Díez

C/ Montserrat, 18 - 6ํㅡ (Eirís)

15009 La Coruña

(Trabajo recibido el 19 mayo de 2003) 